Ebisu

Études japonaises Études japonaises

50 | automne-hiver 2013

Création et valeurs dans le Japon moderne

\title{
Revaloriser la calligraphie : le rôle des expositions pendant les ères Meiji et Taishō
}

書の再評価一明治・大正における展覧会の役割を中心に

Reappraising Calligraphy: the Role of Exhibitions in Meiji and Taishō Japan

\section{Laïli Dor}

\section{OpenEdition}

\section{Journals}

Édition électronique

URL : http://journals.openedition.org/ebisu/1154

DOI : 10.4000/ebisu. 1154

ISSN : 2189-1893

Éditeur :

Institut français de recherche sur le Japon (UMIFRE 19 MAEE-CNRS), Maison franco-japonaise

Édition imprimée

Date de publication : 1 octobre 2013

Pagination : 135-159

ISSN : $1340-3656$

Référence électronique

Lailli Dor, «Revaloriser la calligraphie : le rôle des expositions pendant les ères Meiji et Taishō », Ebisu [En ligne], 50 | automne-hiver 2013, mis en ligne le 21 juin 2014, consulté le 30 avril 2019. URL : http:// journals.openedition.org/ebisu/1154; DOI : 10.4000/ebisu.1154 


\section{Revaloriser la calligraphie Le rôle des expositions pendant les ères Meiji et Taishō}

Laïli DOR*

L'ouverture du Japon à l'Occident entraîna dans le domaine des arts, comme dans bien d'autres, une petite révolution conceptuelle. Parmi les exemples fréquemment cités : l'invention du mot bijutsu 美術 (littéralement " technique du beau "), dont la première occurrence est attestée dans un document de $1871^{1}$ pour la préparation de l'envoi japonais à l'Exposition universelle de Vienne. Derrière ces questions de terminologie se cachaient des remises en question plus profondes : des philosophes japonais comme Nishi Amane 西周, qui publia en 1877 Bimyoggaku setsu 美妙学説 (Théorie de l'esthétique), ou Nakae Chōmin 中江兆民, qui traduisit l'année suivante l'Esthétique d'Eugène Véron, se mirent en effet en devoir de repenser l'art japonais selon des catégories empruntées à l'Occident.

Naoteru Ueno (1958:9) souligne les bouleversements qui en résultèrent dans la conception du champ artistique : un Japonais d'avant l'ère Meiji n'aurait pas vu de lien entre le travail d'un peintre, celui d'un sculpteur

* INALCO, Centre d'études japonaises.

1. Date avancée par Yoshida Chizuko (2011). Sur l'origine du terme bijutsu, voir Kitazawa Noriaki (2010), qui rappelle que la section où exposait le Japon s'intitulait "Darstellung der Wirksamkeit der Kunstgewerbe-Museen » (Représentation de l'activité des musées d'arts appliqués), et que bijutsu constitue probablement une traduction littérale du mot allemand Kunstgewerbe (Kunst $=$ art $=$ 美 $;$ gewerbe $=$ technique, industrie = 術), lequel désigne toutefois les arts appliqués, et non les beaux-arts (Schöne Kunst). 
d'effigies bouddhiques et celui d'un architecte ; il aurait trouvé aberrant de les regrouper dans un même domaine (dénommé " art »). À l'inverse, il aurait perçu la peinture et la calligraphie comme une seule et unique activité, désignée du terme de shoga 書画, là où nous voyons aujourd'hui deux pratiques distinctes.

Dans la hiérarchie des arts qui se mit progressivement en place à compter des années 1870, les beaux-arts, en particulier la peinture et la sculpture, trônaient désormais au sommet. L'estampe et l'artisanat d'art, plébiscités par les collectionneurs occidentaux, tiraient malgré tout leur épingle du jeu. La calligraphie, en revanche, qui ne correspondait pas à un domaine artistique identifié comme tel en Occident, peina à trouver sa place dans le panorama d'ensemble.

Nous aimerions ici nous interroger sur les conséquences à la fois théoriques et pratiques de cette situation, en nous concentrant particulièrement sur la période 1880-1930. C'est en effet au début des années 1880 que la place de la calligraphie dans le monde de l'art fut le plus radicalement remise en question. Pour quelle raison la calligraphie fut-elle écartée du champ des beaux-arts alors que la peinture, dont elle était jusque-là indissociable, y était admise sans discussion ? La calligraphie fut-elle considérée comme simplement extérieure aux beaux-arts, ou comme étant d'une nature inférieure ? Quelles furent, pour les calligraphes, les conséquences de cette mise à l'écart ? Quelles stratégies élaborèrent-ils pour éviter une possible dévaluation de leur activité ?

Nous traiterons la question en deux temps : à une dévalorisation théorique de la calligraphie émanant de critiques extérieurs, les calligraphes répondirent en effet de façon pragmatique par la mise en place d'un circuit de diffusion indépendant - que nous étudierons ici à travers le cas des expositions - qui garantit à terme la survie, et même la prospérité de leur art.

\section{Marginalisation progressive de la calligraphie}

\subsection{Calligraphie et beaux-arts, chronique d'une séparation}

Dans les années 1870, alors qu'avaient lieu les premiers efforts de définition de l'esthétique inspirée de l'Occident, la calligraphie fut, à l'origine, incluse parmi les beaux-arts. La première Exposition nationale pour l'encouragement de l'industrie (Naikoku kangyō hakurankai 内国钊業博覧会), 
en 1877, comportait ainsi une section intitulée "Calligraphie et peinture » (shoga), qui couronna le calligraphe Naruse Taiiki 成瀬大域 (1827-1902). Cette section était également présente lors de la deuxième exposition en 1881, où la peinture à l'huile (abura-e 油絵) se trouvait simplement séparée des autres genres regroupés sous la dénomination "Calligraphies et peintures diverses " (kakushu no shoga 各種の書画). Parmi les calligraphes primés, on comptait Chō Sanshū 長三洲 (1833-1922) et Kusakabe Meikaku 日下部 鳴鶴 (1838-1922), deux figures phares de leur domaine.

Du côté des œuvres classiques, la Société de l'Étang du Dragon (Ryūchikai 龍池会) fournit un bon exemple. Fondée en 1879 par Sano Tsunetami 佐 野常民 (1822-1902), avant de devenir en 1887 l'Association des beaux-arts du Japon (Nihon bijutsu kyōkai 日本美術協会), cette société était destinée à la conservation et à la promotion d'œuvres anciennes du patrimoine japonais. Le règlement fondateur définissait ainsi ses champs d'intérêt, parmi lesquels la calligraphie figurait en bonne place (Urasaki 1974: 98) :

\section{第二十条 本会二於テ調理スル美術ノ区部ハ凡ソ左ノ如シ}

画、書、彫刻、陶器、七宝、漆器、嵌木、紼織、銅器、建築、園治、但便宜部中 ヨ細別スルコトアル

Article 20 Dans le cadre de la présente société seront étudiés les arts appartenant aux catégories suivantes :

Peinture, calligraphie, sculpture, céramique, cloisonné, laque, marqueterie, broderie, orfèvrerie, architecture, art des jardins. Pour des raisons de commodité, des distinctions pourront être effectuées au sein de chaque section ${ }^{2}$.

Si la calligraphie était au départ bien présente dans les expositions et les salons artistiques, comme dans les préoccupations des sociétés d'art, la tendance s'inversa radicalement dans le courant des années 1880. Lors de la troisième Exposition nationale pour l'encouragement de l'industrie, en 1890, la calligraphie était séparée de la peinture et incluse dans une section hétéroclite, "Gravures, photographies et calligraphies diverses " (kakushu han shashin oyobi sho rui 各種版写真及書類). Le jury comptait certes encore une figure de marque en la personne de Chō Sanshū (Andō 1964 : 38), mais cette situation marginale de la calligraphie, qui perdura pour la quatrième

2. Sauf indication contraire, les traductions dans le présent article sont de l'auteure. 
exposition en 1895, n'était que le prélude à sa disparition totale lors de la cinquième et dernière exposition en 1903. La situation fut encore plus simple lors de la mise en place de l'Exposition des beaux-arts du ministère de l'Instruction publique (Monbushō bijutsu tenran-kai 文部省美術展覧会, abrégé Bunten 文展) en 1907 : la calligraphie en fut exclue dès le départ.

Même constat du côté de la Société de l'Étang du Dragon. Peut-être la disparition fut-elle plus rapide encore, puisqu'un article d'Ōuchi Seiran 大内青巒 (1845-1918), paru en 1883 dans le premier numéro du DaiNippon bijutsu shinpō 大日本美術新報 (Bulletin artistique du Grand Japon) et récapitulant les centres d'intérêt de la Société de l'Étang du Dragon, ne mentionne déjà plus la calligraphie. Même si celle-ci resta un temps présente dans les œuvres examinées lors des réunions de l'association, sa place décrut régulièrement.

\subsection{Tentative d'explication}

Comment expliquer ce brusque revirement ? L'historien d'art Satō Dōshin y voit une raison théorique : l'appréhension du domaine de la peinture - jusque-là indissociable de la calligraphie - aurait changé, par un processus de décantation où la peinture serait restée dans le champ des beaux-arts, pendant que la calligraphie en disparaissait. L'année 1882 marque à cet égard un tournant. La revue Tóyō gakugei zasshi 東洋學藝雑誌 publia en effet un échange entre le peintre de l'école occidentale Koyama Shōtarō 小山正太郎 (1857-1916) et le penseur Okakura Tenshin 岡倉天心 (1863-1913), défenseur des arts traditionnels, dont le but affiché était de décider si la calligraphie devait être ou non comptée parmi les beauxarts $^{3}$. Cet échange prit la forme de six articles : les trois premiers furent publiés dans les numéros 8, 9 et 10 (mai, juin et juillet 1882) par Koyama Shōtarō qui affirmait que la calligraphie n'était pas un art, à quoi Okakura Tenshin tenta de répondre dans les numéros 11, 12 et 15 (août, septembre et décembre 1882).

Koyama Shōtarō intitule sa contribution «Sho wa bijutsu narazu » 書八 美術ナラズ (La calligraphie ne fait pas partie des beaux-arts) et y développe l'argumentation suivante : la calligraphie ne répond pas à la caractéristique

3. Pour une étude de ce débat, voir Isabelle Charrier (1999) et Laïli Dor (2013). 
première d'un art, qui est d'être pur de toute visée fonctionnelle. En effet, elle n'a pas pour objectif premier d'être belle, mais de transcrire un discours. Il s'agit avant tout d'une pratique d'écriture, où la dimension esthétique vient se greffer de façon secondaire. De plus, la calligraphie n'a pas, contrairement à la peinture, de dimension mimétique, et ne peut dès lors ni distraire, ni émouvoir par les objets qu'elle représente. Koyama Shōtarō émet même des doutes sur la beauté de certaines calligraphies classiques : ne sont-elles pas plutôt appréciées parce que leurs auteurs sont célèbres (Koyama 1882a : 174)?

Si Koyama Shōtarō pose au départ le problème en termes de définition (la calligraphie appartient-elle ou non au champ des beaux-arts ?), son argumentation glisse rapidement vers un jugement de valeur. Il veut bien admettre, dans certaines conditions très restrictives, une beauté possible de la calligraphie ; mais dans l'ensemble, il s'agit pour lui d'une pratique inférieure aux beaux-arts, et en particulier à la peinture. La calligraphie ne laisse que peu de place à l'inventivité de l'artiste, qui est contraint par l'ordre des mots dans le texte (contrairement au peintre, qui peut disposer à sa guise les différents éléments de l'image). Elle exige donc par définition moins de talent. Par ailleurs, Koyama Shōtarō pointe une dérive selon laquelle les calligraphes de son époque ne font même plus l'effort de donner une expression personnelle aux textes qu'ils écrivent, mais se contentent de copier la manière des grands maîtres du passé (Koyama 1882b : 205 ; 1882 c : 229).

Derrière ces réflexions conceptuelles se cache une raison beaucoup plus terre à terre, dont Koyama Shōtarō ne fait d'ailleurs pas mystère : la faible valeur de la calligraphie sur le marché de l'exportation (Koyama 1882c : 229). Il est vrai que l'Occident, alors en pleine ferveur du japonisme, semble n'avoir éprouvé pour la calligraphie qu'un intérêt modéré. Pour le peintre, la valeur esthétique se trouve en quelque sorte indexée à la valeur marchande : si les collectionneurs occidentaux, en qui il voit les arbitres suprêmes des questions esthétiques, ne se préoccupent pas davantage d'acheter de la calligraphie, c'est que cet art n'en vaut pas la peine. Il ne s'agit pas là d'une simple excentricité de l'auteur : Chelsea Foxwell (2012 : 146-147) rappelle à quel point le rapport entre valeur esthétique et enjeux économiques était étroit dans le Japon des années 1880, où le gouvernement se trouvait confronté au défi paradoxal de promouvoir l'exportation d'œuvres pour exploiter au mieux la vogue du japonisme en Occident, tout en ralentissant la fuite des chefs-d'œuvre du passé. 
Koyama Shōtarō pousse même plus loin le raisonnement, en faisant intervenir, comme horizon ultime de sa réflexion, la question de la valeur intellectuelle et morale. Il commence par accuser à mots couverts les Japonais de naïveté. Affirmer que la calligraphie est un art alors que l'Occident, seul juge en la matière, considère ce domaine comme négligeable, ne peut qu'exposer le Japon au ridicule. Inclure la calligraphie dans la section artistique des expositions universelles invitait nécessairement le public à établir une comparaison défavorable avec la peinture (Koyama 1882c : 229). Les Japonais passeraient alors pour des ignorants incapables de comprendre ce qu'est l'art. Or, pour Koyama Shōtarō, il n'y aurait pas là seulement de l'aveuglement, mais bien pire, une forme de mauvaise foi : ce serait une véritable tromperie, de la part des organisateurs, que de vouloir faire passer la calligraphie pour un art.

\subsection{Fenollosa et le « Discours sur la vraie théorie des arts»}

La rhétorique de Koyama Shōtarō renvoie en fait, de façon implicite, à celle d'Ernest Fenollosa et notamment aux arguments par lesquels il discrédite la peinture de lettrés dans son Bijutsu shinsetsu 美術真説 ${ }^{4}$ (Discours sur la vraie théorie des arts), prononcé au Musée de l'éducation du parc d'Ueno en mai 1882, soit quelques mois avant la controverse entre Koyama et Okakura.

En apparence, Koyama Shōtarō s'inscrit pourtant en faux contre Fenollosa, qu'il attaque en termes à peine voilés lorsqu'il s'en prend, dans son premier article, à la Société de l'Étang du Dragon, celle-là même qui avait organisé le discours. Même l'influence de Fenollosa est objet de moquerie, lorsque Koyama raille, dans son troisième article, le brusque revirement des esthètes japonais qui se passionnèrent pour la peinture moderne aux pigments traditionnels (nihonga 日本画) sitôt qu'ils entendirent un Occidental en faire l'éloge.

Pourtant, Koyama Shōtarō défend, au fond, une position étrangement similaire à celle de l'Américain qui écartait du champ artistique la calligraphie et la peinture de lettrés. Le principal grief de Fenollosa envers la peinture de lettrés était son caractère impropre à l'exportation. Il motivait ce

4. Pour une analyse plus précise du texte de Fenollosa, voir Arthur Mitteau (2013a). 
jugement par des considérations esthétiques : ce genre de peinture ne saurait être tenu pour un art visuel à part entière, car son inspiration première est littéraire et non picturale. Dès lors, la peinture de lettrés ne pouvait manifester l'unité de sujet qui constituait selon lui l'un des critères incontournables dans la définition des arts. Or tels se trouvent être précisément les arguments employés par Koyama Shōtarō pour discréditer la calligraphie. De même, lorsque Koyama Shōtarō évoque la réalité japonaise de son époque, c'est pour souligner son infériorité par rapport à la Chine, un argument déjà utilisé par Fenollosa : le développement de la calligraphie en tant que discipline artistique ne peut produire au mieux que des copistes, qui n'égaleront jamais les maîtres chinois du passé.

\subsection{La réponse d'Okakura Tenshin : une réhabilitation incomplète}

La réponse d'Okakura Tenshin, survenue dès la fin de l'année 1882, aurait dû contribuer à réhabiliter la calligraphie, mais le débat s'avère en fait largement biaisé (Dor 2013 : 294-295). Cette dimension est évidente dès le titre de la réponse d'Okakura Tenshin, " "Sho wa bijutsu narazu" no ron o yomu »書ハ美術ナラズ』/論习読ム (Lecture de l'essai “La calligraphie ne fait pas partie des beaux-arts") : plutôt que de postuler une assertion inverse à celle de Koyama Shōtarō, en affirmant que la calligraphie fait partie des beaux-arts, l'auteur adopte une position en retrait. Une lecture attentive laisse supposer, avant tout, une confrontation personnelle où Okakura Tenshin, disciple de Fenollosa et grand défenseur de l'art japonais, voit dans Koyama Shōtarō avant tout l'incarnation du camp adverse, à la solde de l'Occident.

Dans le débat, la grande priorité d'Okakura n'est donc pas tant de démontrer que la calligraphie appartient bien au domaine des beaux-arts, que de prouver que son adversaire a tort. Même dans cette perspective, Okakura Tenshin esquive régulièrement la confrontation. Ainsi, dans son troisième article, il discute les opinions énoncées par Koyama Shōtarō sur le rapport entre la sculpture et la gravure, puis sa définition de la peinture et de ses objectifs, mais il n'est au fond que fort peu question de calligraphie. L'attitude ambiguë d'Okakura vis-à-vis de la calligraphie se trouve confirmée par le fait que, une fois devenu directeur de l'École des beaux-arts de Tokyo (Tōkyō bijutsu gakkō 東京美術学校) en 1889, il ne fit rien pour y promouvoir un enseignement de la calligraphie. 


\subsection{Conséquences théoriques}

Après la réponse d'Okakura Tenshin, plus personne ne semble avoir directement pris à partie l'un des intervenants. Ce débat théorique, le premier à examiner la nature artistique de la calligraphie, induit donc une situation de flottement : d'une part, il déboucha sur une aporie, et la question n'est pas tranchée de manière nette; d'autre part, c'est une controverse sans écho immédiat. Les deux protagonistes, qui n'étaient pas eux-mêmes calligraphes, n'ont par la suite accordé que peu d'intérêt au sujet, et il fallut attendre près de vingt ans avant qu'un article aborde à nouveau la question de la calligraphie et des beaux-arts. Malgré ce décalage chronologique, les interventions ultérieures furent lourdement marquées par l'héritage de la controverse initiale : presque toutes furent en effet dictées par une volonté de réhabiliter la calligraphie en prouvant qu'elle n'était pas inférieure aux beaux-arts.

La première réaction connue à la controverse est l'article de l'historien Kawada Takeshi 川田剛 (1830-1896), intitulé «Sho wa bijutsu taru no setsu » 書は美術たるの説 (Pourquoi la calligraphie est un des beaux-arts) (Kawada 1892), publié plus de dix ans après le débat. Kawada Takeshi, savant confucéen, n'était pas davantage calligraphe que les protagonistes du débat initial, mais il entendit bien tenir le rôle qui n'avait été qu'imparfaitement rempli par Okakura Tenshin, en apportant cette fois une contradiction explicite à la position de Koyama Shōtarō. Certes, sa démarche n'est pas exempte des problèmes méthodologiques qui pesaient déjà sur la controverse. De même que Koyama Shōtarō, tout en affirmant une démarche déductive, se contentait souvent d'énumérer au lieu d'argumenter, Kawada Takeshi n'échappe pas au descriptif : sur les quelques trente-cinq pages que compte son article, seule une petite dizaine sont véritablement consacrées à la discussion esthétique. Le reste consiste en un panorama historique de la calligraphie en Chine et au Japon - panorama où les questions de valeur se lisent néanmoins en filigrane. Là où Koyama Shōtarō et Okakura Tenshin acceptaient sans discussion la suprématie de l'héritage chinois, Kawada Takeshi défendit au contraire la spécificité du patrimoine japonais :

\section{されば、書は支那の美術にして我國の美術に非ずと思ひ誤る人多かれど、さには 非ず}

En conséquence [de la riche histoire de la calligraphie en Chine], nombreux sont ceux qui considèrent à tort la calligraphie comme un art propre à la Chine, et non à notre pays, mais tel n'est pas le cas. (Kawada 1892 : 360) 
En 1926, l'historien d'art Taki Seiichi 瀧精一 (1873-1945) rédigea à son tour - sous son nom de plume de Setsuan 拙庵 - un article intitulé «Shodō to bijutsu » 書道と美術 (La calligraphie et les beaux-arts) (Taki 1926), l'un des très rares textes que la prestigieuse revue artistique Kokka 國華 ait consacrés à la calligraphie. L'aspect argumentatif est plus travaillé, l'auteur adoptant une réflexion générale sur la calligraphie plutôt que de dresser une liste d'exemples historiques. Il y propose une interprétation du rapport entre calligraphie, écriture et beaux-arts :

成程西洋には書を上手に書く事を風習は全くないことはないが、それを絵画䧓刻 その他の美術の例に加へて尊崇すると云ふ事は更にない。

Bien sûr, cela ne signifie pas qu'il n'y ait en Occident aucune habitude de pratiquer la belle écriture, mais celle-ci n'est pas particulièrement révérée à l'égal de la peinture, de la sculpture ou d'autres exemples des beaux-arts. (Taki $1926: 28$ )

Pour Taki, la hiérarchie entre les arts ne vient pas de leur qualité intrinsèque, mais d'une échelle de valeurs établie de l'extérieur (l'écriture n'est pas moins belle, elle est juste moins prisée que la peinture). Le calligraphe Onoe Saishū 尾上柴舟 (1876-1957) va plus loin, dans sa préface à l'histoire de la calligraphie par Okuyama Kindō 奥山錦洞 (Onoe 1927) :

東洋芸術と線とは、不離の関係にあると、常に私は思ふ。色彩のみで効果を上げ てみるのもあるが、それはむしろ少なく、線の力、色、匂によつて、不朽の名品 の多くが作られてるる。

線の、最も単純に用みられて出来る東洋芸術は書である。東洋以外の国の文字 も線で成立つ。しかし、現はされた高踏的情趣は、これに豊かで、彼に乏しい。 彼のは、ただに文字である。書といふ一派の芸術は確に東洋にのみ存在するので ある。

J'ai toujours pensé que les arts de l'Orient entretenaient avec la ligne un lien indissociable. Bien sûr, il existe des œuvres qui tirent tout leur effet de la seule couleur, mais elles sont plutôt rares, alors que les œuvres immortelles qui tirent leur force de la ligne et leur chatoiement de la couleur sont au contraire nombreuses.

L'art oriental qui fait la plus simple utilisation de la ligne est la calligraphie. Dans les pays extérieurs à l'Orient, l'écriture fait également appel à la ligne. Mais l'émotion raffinée qui s'y trouve exprimée est riche chez nous et pauvre chez eux. Leurs écritures ne sont que des lettres. Parmi les arts, celui que l'on appelle la calligraphie n'existe qu'en Orient. 
Bien qu'Onoe ne cite pas explicitement la controverse, sa vision de la calligraphie comme un art de l'écriture, ou plutôt comme une impondérable valeur ajoutée, en termes esthétiques, qui vient métamorphoser l'écriture en art, renvoie très clairement à l'argument de départ de Koyama Shōtarō. Bien qu'il aboutisse à des conclusions diamétralement opposées à celles de son prédécesseur, Onoe Saishū a recours à un procédé similaire, à savoir le glissement d'une question de définition vers un jugement de valeur. Il part ainsi d'une différence de nature (l'opposition entre les arts de l'Orient, caractérisés par le primat de la ligne, et ceux de l'Occident, présentés implicitement comme le domaine de la couleur) pour établir le caractère particulièrement représentatif de la calligraphie parmi les arts orientaux. Dans le deuxième paragraphe, il change brusquement les prémisses : la comparaison ne s'effectue plus avec la peinture, ou avec d'autres arts, mais avec l'écriture de l'Occident, ce qui lui permet d'affirmer l'infinie supériorité de la calligraphie orientale.

La réponse théorique au débat Koyama-Okakura fut, à maints points de vue, peu satisfaisante. Non seulement personne ne parvint à (ou ne voulut ?) apporter une contradiction convaincante aux arguments de Koyama Shōtarō selon lesquels la calligraphie n'était pas un art, mais l'ombre de la controverse sembla même obérer durablement le discours théorique ultérieur. En effet, les théoriciens eurent tendance à penser la calligraphie dans une perspective de réhabilitation, plutôt que de définition.

Plus curieux encore fut le silence des calligraphes qui, en dehors d'Onoe Saishū (dont l'intervention est tardive) et de quelques rares exceptions, ne s'impliquèrent guère dans le débat. Que la contradiction, en 1882, ait été apportée à Koyama Shōtarō par un théoricien extérieur au monde de la calligraphie est déjà surprenant. Chō Sanshū et Kusakabe Meikaku, les deux calligraphes primés lors de l'Exposition nationale pour l'encouragement de l'industrie de 1881 - celle-là même à laquelle s'en prenait Koyama Shōtarō dans son premier article - auraient constitué des interlocuteurs tout naturels. Rappelons toutefois que Chō Sanshū, qui défendait avant tout la place de la calligraphie dans le système scolaire, ne pouvait qu'approuver les positions de Koyama Shōtarō lorsqu'il affirmait, en conclusion de son troisième article, que la calligraphie devait être enseignée comme une matière scolaire apparentée à l'écriture, et non comme un art.

L'opinion de Kusakabe Meikaku eût certainement été intéressante, car ses positions concernant le rapport entre la calligraphie et les beaux-arts 
étaient diamétralement opposées à celles de Koyama Shōtarō : la calligraphie, art millénaire comptant parmi les «six arts » (rikugei 六藝) de la Chine ancienne, était pour lui infiniment supérieure à une activité d'artisan comme la peinture, et aux beaux-arts récemment importés d'Occident. De fait, le rapprochement entre le monde de la calligraphie et celui des beauxarts ne s'amorça, bien timidement, qu’après la mort de Kusakabe Meikaku, lorsque la position de prééminence sur la scène calligraphique fut reprise par un calligraphe du courant adverse, Bundō Shunkai 豊道春海 (18781970), qui prônait au contraire un esprit d'ouverture.

Que penser de ce peu d'empressement des calligraphes à défendre la valeur de leur champ d'activité, menacé dans les faits par la prééminence des beaux-arts au sens occidental ? Leur silence dans les débats théoriques se trouve en fait compensé par une forte activité sur le terrain. Plutôt que de s'engager dans une confrontation avec les artistes, ils se mirent en devoir de constituer un circuit professionnel indépendant ; plutôt que d'affirmer, sur le papier, une égalité de valeur entre calligraphie et beaux-arts, ils constituèrent des réseaux qui leur offrirent une visibilité comparable à celle dont bénéficiaient, par exemple, les peintres. Bref, ils refusèrent de s'insérer dans une hiérarchie de valeurs dictée par les artistes issus des beaux-arts, où la calligraphie aurait nécessairement occupé une position marginale, pour mettre au contraire en place un système obéissant à des critères d'évaluation qui leur soient propres.

\section{Réhabilitation de la calligraphie : le rôle des expositions}

Face au peu d'empressement du gouvernement de Meiji à mettre en place des circuits de formation et de vente dédiés à la calligraphie, les calligraphes formèrent leurs propres réseaux, dont la forme la plus aboutie fut l'association $(k a i \text { 会 })^{5}$. Parmi les multiples activités de ces structures, telles

5. Il s'agit là, au demeurant, d'un processus assez similaire à celui que décrit Nathalie Heinich dans l'Occident du XIX ${ }^{e}$ siècle, où le vide institutionnel lié à la fin du système académique, survenant au moment même où la population des artistes connaissait un accroissement conséquent, aboutit à la formation de groupes qui avaient pour objectif de se substituer à l'Académie (Heinich 1996 : 49). 
que l'enseignement, la recherche, ou la publication de revues, nous nous concentrerons ici sur un aspect particulier, celui des expositions. Sans doute est-ce cet aspect qui permit aux calligraphes d'assurer le plus concrètement la visibilité de leur art et la diffusion de leurs ouvres, évitant ainsi que la calligraphie ne soit reléguée aux marges de la scène artistique.

\subsection{Les réunions de lettrés}

Les regroupements de calligraphes n'apparurent pas brutalement à l'ère Meiji, mais vinrent plutôt réactiver des formes d'organisation plus anciennes. Ainsi, il existait déjà un système de diffusion des œuvres riche d'une longue histoire, les réunions de lettrés, ou gakai 雅会, qui pouvaient se décliner en réunions de thé (chakai 茶会), réunions de poésie (shikai 詩会), ou réunions de calligraphie et de peinture (shogakai 書画会). Dans ce dernier type de réunions, l'organisateur présentait non seulement les œuvres de sa collection, mais aussi occasionnellement celles qu'il avait pu exécuter lui-même, ou encore celles d'un invité. Il pouvait arriver qu'il expose des instruments de peinture ou de calligraphie, lorsqu'il s'agissait de pièces rares. Ce mode de rencontre semi-privée, qui permettait à la fois le contact entre artistes et la diffusion des œuvres, permit de pallier sans rupture la période où la calligraphie était exclue des salons.

L'historien de la calligraphie Kondō Kōshi décrit comme suit le déroulement d'une réunion de lettrés (Kondō 1991 : 191) : les invités - en général une dizaine - étaient accueillis dans le jardin, puis se rendaient dans la pièce choisie pour la réunion. On apportait depuis la réserve les œuvres et les pièces de collection destinées à l'appréciation des convives, et la discussion s'engageait. Ensuite, un repas était servi, suivi d'une séance de peinture ou de composition de poèmes.

Ce mode de fonctionnement appelle quelques remarques. Premièrement, la sélection des œuvres était délibérément subjective, dictée par les goûts de l'organisateur. Deuxièmement, la notion de public se trouvait restreinte au petit cercle des convives. L'avantage résidait dans la possibilité d'un contact direct entre l'artiste et ses admirateurs, lesquels se voyaient par ailleurs offrir une chance de contempler l'œuvre en cours de création. Bien plus, la distinction entre artiste et public se trouvait brouillée, puisque les participants, après avoir contemplé des œuvres anciennes ou la démonstration d'un maître contemporain, prenaient à leur tour le pinceau. Création, critique 
et appréciation allaient alors de pair et faisaient intervenir indifféremment calligraphie, peinture et poésie.

Ces réunions de lettrés ne disparurent pas avec l'entrée du Japon dans l'âge moderne, mais leur forme évolua. Le changement le plus remarquable fut sans doute l'augmentation du nombre des participants, qui atteignit parfois plusieurs centaines. Les réunions ne furent alors plus organisées chez un hôte, mais dans un restaurant, et les établissements spécialisés dans l'accueil de ces manifestations devinrent célèbres, comme le Nakamura-rō 中村楼 dans le quartier de Ryōgoku à Tokyo.

\subsection{Valeur marchande : des réunions de lettrés aux kōkankai 交換会}

La calligraphie étant exclue des salons qui, par le biais des prix et des commandes, offraient aux artistes une source de revenus, les réunions de lettrés évoluèrent pour devenir un lieu non seulement de création, mais aussi de vente. Dans le courant des années 1870, l'entrée devint payante, avec des frais de 5 à 15 sen qui garantissaient l'accès pour toute la journée. De même, les œuvres exécutées par les lettrés et les calligraphes invités, qui avaient jusque-là été distribuées gratuitement aux participants, furent désormais proposées à la vente. Les réunions prirent donc une nouvelle dimension, et leur fonctionnement mériterait sans doute un rapprochement avec celui des ventes par adjudication évoquées par Cléa Patin ${ }^{6}$. Elle cite ainsi une évocation des réunions de marchands, dont la description pourrait s’appliquer à l'identique aux réunions de lettrés de l'ère Meiji :

Autrefois, ils se réunissaient dans des restaurants ou des auberges, où ils exposaient des peintres. Ils mangeaient, buvaient, puis s'achetaient mutuellement des œuvres ${ }^{7}$.

Les ventes par adjudication constituèrent donc un prolongement naturel des réunions de lettrés. Citons ici à titre d'exemple un encart paru dans la revue Shodō 書道 en juillet 1938 - soit à une date un peu plus tardive, il

6. Voir son article « Le système des ventes d'œuvres d'art réservées aux professionnels au Japon (kōkankai) » dans le présent dossier (Patin 2013).

7. Entretien avec la galerie Kawafune ギャラリー川舟, le 27 novembre 2010 (Patin $2013: 191)$. 
est vrai, que la période considérée dans le cadre du présent article. Le texte s'intitule « Dai ikkai Shumi no kōkankai : keika hōkoku » 第一回趣味の交 換會一一經過報告 (Compte rendu de la première vente par adjudication des connaisseurs), et donne la liste des ouvres et objets mis en vente : 73 rouleaux de calligraphie et de peinture (shogafuku 書画幅), 34 encriers anciens (koken 古硯), 14 livrets de calligraphie ancienne (kohōjō 古法帖), 28 verseuses à eau (suiteki 水摘) et 98 objets de nature diverse (matériel pour la gravure des sceaux, bâtons ou pains d'encre anciens, etc.). Ce type de vente perpétua un espace d'appréciation où se trouvaient juxtaposés, comme dans les réunions de lettrés, œuvres (anciennes et modernes) et instruments (Shodō 1938 : 51). Dans le domaine purement artistique, à l'inverse, le mode d'exposition évolua dans le sens d'une différenciation accrue.

\subsection{Valeur esthétique : des réunions de lettrés aux expositions}

Jusqu'à la fin des années 1880 , les réunions de lettrés conservèrent toute leur importance, et l'on peut même dire que leur pouvoir de diffusion s'accrut en proportion du public qu' elles attiraient. Mais ce système comportait également des inconvénients, au premier rang desquels son inégalité d'accès. Les réunions ayant lieu dans les grandes villes (principalement Tokyo et Kyoto), les calligraphes qui se trouvaient exercer leur activité ailleurs étaient défavorisés. À l'inverse, ceux qui habitaient Tokyo bénéficiaient d'un avantage incomparable, que ne pouvaient même concurrencer ceux de Kyoto. Sans doute ce point contribua-t-il d'ailleurs à renforcer la prééminence de la nouvelle capitale comme centre de l'activité calligraphique.

De même, les réunions de lettrés représentaient, au début de l'ère Meiji, un excellent tremplin pour les jeunes talents. Or, à partir du moment où ces réunions permirent aux calligraphes de gagner leur vie en faisant payer l'exécution des œuvres, certains artistes devinrent des habitués de ce genre de performances publiques, et monopolisèrent une place qui aurait dû revenir à des artistes plus jeunes ou moins connus.

Un dernier problème concernait le caractère subjectif de la sélection, qui nuisait à l'image des réunions de lettrés comme garantes de la valeur esthétique d'une œuvre. Les œuvres, ou les maîtres invités, étaient en effet choisis en fonction des goûts et des affinités de l'organisateur. Ces réunions constituaient un circuit fermé, où la participation d'un calligraphe dépendait moins de son talent que de son réseau de connaissances. 
À la fin de l'ère Meiji, les réunions de lettrés avaient perdu beaucoup de leur crédibilité, et les calligraphes se mirent en quête d'autres modes d'exposition, offrant cette fois des garanties sur la valeur technique et artistique des œuvres exposées. Le modèle qui s'imposa à eux fut celui de l'exposition publique avec jury (celle que désigne le terme tenrankai 展覧会), fort proche finalement des structures dont ils s'étaient trouvés exclus dans le courant des années 1880. Les réunions de lettrés ne disparurent pas, pour autant, du jour au lendemain. Ainsi, à l'ère Taishō encore, la Réunion du Pavillon des Orchidées de Taishō (Taishō rantei kai 大正蘭亭会), exposition et séance de poésie autour de l'œuvre de Wang Xizhi 王羲之 (303-361), organisée en 1913 par le Club des Arts de Tokyo (Tōkyō bijutsu kurabu 東京美術俱楽部) et le Club de Nihonbashi (日本橋俱楽部), connut un grand succès. Il s'agit malgré tout du dernier exemple véritablement marquant, et le modèle dominant devint celui des expositions, où les œuvres modernes étaient séparées des classiques, et où les instruments, sans parler du processus de création lui-même, avaient disparu.

\subsubsection{Les expositions d'associations}

L'organisation d'expositions publiques s'orienta dans deux directions différentes. La première visait à créer des expositions qui soient spécifiques à la calligraphie, en général dans le cadre d'une association, avec une sélection des œuvres par un jury interne.

La première véritable tentative ${ }^{8}$ eut lieu en 1890 , sous l'impulsion du linguiste et critique Ōtsuki Joden 大槻如電 (1845-1931). Érudit et intéressé par les questions linguistiques, puisqu'il participa à la rédaction du Nouveau dictionnaire sélectif des caractères chinois (Shinsen jisho 新撰字書) ${ }^{9}$, rien n'indique toutefois qu'il ait jamais pratiqué ou enseigné la calligraphie. Son intérêt pour le domaine est même surprenant, puisque ses posi-

8. En 1874, une exposition intitulée "Exposition de calligraphies et de peintures du temple de Shōheizaka » (Seidō Shōheizaka shoga ten 聖堂昌平坂書画展) constitua un premier exemple d'exposition publique, mais elle était encore partagée entre peintures et calligraphies.

9. Ce dictionnaire, publié en 1872 par le ministère de l'Instruction publique, constitua l'un des premiers efforts officiels pour limiter et rationaliser l'usage des caractères chinois, notamment dans l'éducation. 
tions linguistiques le portaient davantage vers une restriction, voire un abandon, de l'usage des kanji.

Ōtsuki Joden évoque ses souvenirs de l'exposition dans un article paru en 1911 dans le numéro inaugural de la revue Shoen 書苑 (Le Jardin de la calligraphie). Animé d'un esprit pionnier, il souhaitait organiser la première exposition intégralement consacrée à la calligraphie. Notant le succès des expositions de peinture, succès qu'il attribuait à l'influence d'Ernest Fenollosa, Ōtsuki déplorait qu'il n'existe pas le moindre pendant dans le domaine de la calligraphie - une aberration culturelle, puisque la calligraphie était à ses yeux beaucoup plus représentative des arts d'ExtrêmeOrient que la peinture. Son vœu le plus cher était donc de susciter, chez ses contemporains, un enthousiasme pour la calligraphie égal à celui que rencontrait la peinture de type nihonga, et c'est dans cette perspective qu'il décida d'organiser sa propre exposition.

L'exposition était un projet de vaste envergure, visant à présenter aussi bien des œuvres contemporaines que des trésors classiques. Elle se déroula dans l'annexe du Club des Pairs (Kazoku kaikan 華族会館) au parc d'Ueno, lieu qui tenait davantage du club de gentlemen que de l'institution artistique. Les créations des maîtres contemporains se voyaient réserver le rezde-chaussée du bâtiment, cependant que l'étage était consacré aux œuvres classiques. Ōtsuki ne donne sur ces dernières aucune précision de nombre ou de nature, mais utilise à leur propos l'expression kokin naigai 古今内外 qui semble impliquer qu'elles couvraient une période historique large, et qu'elles comptaient à la fois des pièces chinoises et japonaises.

Si le nombre exact de calligraphies exposées n'est pas connu, non plus que la liste exhaustive des participants, Ōtsuki précise qu'une bonne trentaine de calligraphes (sur les 60 à 70 qu'il avait pu répertorier à Tokyo) assistèrent à la réunion préparatoire, et l'exposition finale compta au moins une figure majeure en la personne d'Iwaya Ichiroku 嚴谷一六 (1834-1905).

L'entreprise fut un échec retentissant, rendu plus cuisant encore par la comparaison avec la peinture, puisque se déroulait en même temps une exposition de l'Association des beaux-arts du Japon (Nihon bijutsu kyōkai 日本美術協会) qui attirait les foules pendant que celle d'Ōtsuki restait déserte. Calamiteuse en termes de fréquentation, la manifestation fut également un gouffre financier. Ōtsuki en fut de sa poche pour un montant qu'il évalue entre 70 et 80 yens. Malgré son échec à court terme, l'exposition ouvrit des perspectives pour l'avenir. Il s'agit en effet 
du premier exemple documenté d'une exposition purement consacrée à la calligraphie et qui soit, de surcroît, ouverte au public, par opposition aux réunions de lettrés qui n'étaient accessibles qu'aux seuls invités, si nombreux soient-ils.

C'est également la première exposition adossée à une association, la Société des Six Styles (Rikusho-kai 六書会). La Société des Six Styles n'était, certes, qu'une émanation de l'exposition, et non un cadre de rencontres régulières. Malheureusement, Ōtsuki en dit très peu sur cette structure. Il explique l'avoir fondée dans le but exprès de gérer les préparatifs, et l'association semble avoir été dissoute après l'échec de l'exposition. La démarche était pourtant novatrice. Alors que le mouvement des associations allait prendre une telle ampleur que, trente ans plus tard, il ne serait plus envisageable d'exposer sans passer par une structure de ce type, il n'existait en 1890 qu'une seule association, la Société des Propos détachés du monde (Juppitsuhō-dō seidankai 述筆法堂清談会) du calligraphe Matsuda Sekka 松田雪柯 (1823-1881), et elle ne semble pas avoir organisé d'exposition.

Un autre point intéressant dans la démarche d'Ōtsuki Joden était la volonté de mêler calligraphie ancienne et création contemporaine - une démarche qui s'est perdue très rapidement. Les expositions de calligraphie ancienne furent par la suite prises en charge par les musées, là où les associations préféraient assurer la promotion des œuvres de leurs membres.

Ces associations en effet ne tardèrent pas à comprendre tout le parti qu'elles pouvaient tirer des expositions. La première à s'être risquée dans l'aventure est pratiquement homonyme de celle d'Ōtsuki Joden, qui y fait d'ailleurs allusion dans son article. Dénommée Association des Six Styles (Rikusho kyōkai 六書協会), et dirigée par Watanabe Saō 渡辺沙鷗 (18641916), elle fut fondée en 1901, et organisa, à compter de l'année suivante, une exposition annuelle dans les locaux de l'Association des beaux-arts du Japon. À compter de 1908, une autre association, la Société du Pinceau vigoureux (Kenpitsu-kai 健筆会) fondée par Maeda Mokuhō 前田默鳳 (1853-1918) lui emboîta le pas.

Le vrai tournant intervint grâce à la Société pour la promotion de la calligraphie japonaise (Nihon shodō sakushin kai 日本書道作振会), qui avait pour particularité de rassembler les calligraphes sans considération d'école ou de courant. Dès 1925, l'association organisa sa première exposition, au Club des beaux-arts d'Ueno. Le lieu était trop exigu pour permettre une appréciation correcte des œuvres, de sorte que l'événement ne connut pas 
un retentissement extraordinaire. C'est donc surtout la deuxième exposition qui marqua les esprits, moins peut-être par son contenu lui-même que par les épisodes rocambolesques qui entourèrent son organisation, et qui cristallisèrent les rapports entre calligraphes et artistes issus des beauxarts. Cette exposition eut lieu, en 1926, au Musée des beaux-arts de Tokyo (Tōkyōfu bijutsukan 東京府美術館 ${ }^{10}$ ) récemment ouvert. Le projet initial de cet établissement, conçu à l'instigation du gouvernement municipal de Tokyo, était d'y inclure des expositions de calligraphie, mais ce point souleva un refus catégorique des milieux artistiques, pour qui prévalait encore l'opinion de Koyama Shōtarō selon laquelle la calligraphie n'est pas un art.

Or, Bundō Shunkai avait perçu toutes les possibilités du lieu, et partit donc en croisade pour convaincre les représentants du milieu de la peinture d'autoriser les expositions de calligraphie. Il finit par obtenir gain de cause en allant plaider en personne auprès du mécène dont la fortune avait permis l'édification du musée. Ainsi, le Musée d'Ueno accueillit-il finalement dès son ouverture des expositions de calligraphie ${ }^{11}$.

Cette deuxième exposition de la Société pour la promotion de la calligraphie permet de mesurer le chemin parcouru depuis l'expérience malheureuse de la Société des Six Styles. Bundō Shunkai avait sur Ōtsuki Joden l'avantage d'appartenir lui-même à la scène calligraphique, où il occupait même une position phare en tant que président d'une association qui était alors la plus puissante du pays. Il n'eut donc pas à affronter, comme l'avait fait son prédécesseur, le désintérêt du domaine même qu'il entendait défendre. Bien plus, loin de devoir faire aveu d'infériorité face au monde de la peinture, il put s'offrir le luxe d'aller défier les peintres sur leur territoire, au sens le plus géographique du terme, puisqu'il entendait les concurrencer sur l'occupation d'un lieu d'exposition dont ils souhaitaient s'arroger l'exclusivité.

Si les progrès étaient évidents depuis 1890, les acquis demeuraient fragiles. Le rapprochement, sur un pied d'égalité, avec les milieux des

10. Ce musée est aujourd'hui connu sous le nom de Tōkyōto bijutsukan 東京都美術館. 11. Ceci est resté vrai jusqu'à une époque très récente, puisque ce musée a accueilli les grandes expositions annuelles de calligraphie patronnées par les journaux jusqu’à l'ouverture du Centre national des arts de Tokyo (Kokuritsu shin bijutsukan 国立新美術 館) à Roppongi en 2007. 
beaux-arts, restait bien timide, car l'autorisation avait été conquise de haute lutte, et obtenue par la ruse plutôt que par consensus. La place marginale de la calligraphie se révèle assez clairement si l'on constate que Bundō Shunkai, président d'une association qui était représentative de la communauté des calligraphes dans son ensemble, obtint gain de cause en contournant les représentants des beaux-arts. La réévaluation de la calligraphie par rapport aux beaux-arts n'était donc pas entièrement concluante : si les calligraphes se percevaient désormais comme égaux aux artistes, la réciproque n'était pas vraie.

\subsubsection{Réintégration de la calligraphie dans les expositions artistiques}

En même temps qu'ils organisaient des expositions qui leur soient propres, les calligraphes militaient également pour la réintégration de la calligraphie dans les expositions artistiques et les salons nationaux. L'enjeu y était quelque peu différent de celui des expositions d'associations : il ne s'agissait plus uniquement de distinguer, pour leur excellence, certaines œuvres ou certains calligraphes parmi d'autres, mais de réaffirmer une égalité de valeur entre la calligraphie en tant que domaine, et les beaux-arts.

En 1912, soit cinq ans après l'exclusion du Bunten (Exposition des beaux-arts du ministère de l'Instruction publique), la calligraphie fut finalement incluse dans la section "Beaux-arts " de la deuxième Exposition industrielle de Tokyo (Tōkyō kangyō hakurankai 東京勧業博覧会). Cette exposition marquait un premier pas symbolique, mais l'intégration de la calligraphie aux sections artistiques n'était pas définitivement acquise.

Certes, ce fut à nouveau le cas lors de l'Exposition de Taishō (Taishō hakurankai 大正博覧会) en 1914. La calligraphie y était associée à la gravure de sceaux dans une section intitulée "Calligraphie et gravure de sceaux " (sho to tenkoku 書と管刻), qui constituait à son tour l'une des douze subdivisions de la section "Beaux-arts et artisanat d'art " (bijutsu oyobi bijutsu kōgei 美術及び美術工芸). Pour chaque section de l'exposition, un concours avait été lancé à l'échelle nationale, et un jury était chargé de sélectionner les œuvres retenues. La calligraphie et la gravure de sceaux, bien que réunies dans une même section, faisaient chacune l'objet d'un jury séparé. Le jury de calligraphie incluait plusieurs figures importantes, telles que Nishikawa Shundō 西川春洞 (1847-1915) et Ono Gadō 
小野鶖堂 (1862-1922) pour la calligraphie en kanji, et Ōguchi Shūgyo 大口周魚 (1864-1920) pour celle exécutée en kana. La gravure des sceaux était quant à elle évaluée par Imaizumi Yūsaku 今泉雄作 (1850-1931), Okamura Baiken 岡村梅軒 (1863-1920) et Ashino Nanzan 芦野楠山.

Les calligraphes récompensés étaient relativement jeunes : Bundō Shunkai (35 ans) avait obtenu la plus haute distinction pour les kanji, cependant que la calligraphie en kana était représentée par Onoe Saishū (37 ans) et Katō Kyokurei 加藤旭嶺 (1870-1960) (44 ans), qui fonda plus tard à Kyoto la Société calligraphique de Heian (Heian shodō-kai 平安書道会). L'exposition avait donc parfaitement rempli son rôle de découverte et de promotion des talents : si les membres du jury étaient des calligraphes reconnus de l'ère Taishō, les primés jouèrent plutôt un rôle majeur après la Deuxième Guerre mondiale (Uozumi 2001 : 114). De plus, la compétition à l'échelle du pays entier, au-delà des distinctions d'école ou de chapelle, fit beaucoup pour sceller la dimension nationale de la calligraphie.

En 1922, grâce au soutien de la Société japonaise de calligraphie (Nihon shodōkai 日本書道会), la calligraphie fut incluse dans l'Exposition de Tokyo pour la célébration de la paix (Heiwa kinen Tōkyō hakurankai 平和記念東京博覧会). Cette exposition, tout en confirmant la place de la calligraphie dans les grandes expositions nationales, fut peut-être moins marquante que les deux précédentes. Contrairement à l'Exposition industrielle de Tokyo et à l'Exposition de Taishō, la calligraphie ne fut pas incluse dans la section des beaux-arts. Le Rapport administratif de l'Exposition de Tokyo pour la célébration de la paix (Heiwa kinen Tökyō hakurankai jimu hōkoku 平和記念東京博覧会事務報告) comporte en effet la note suivante concernant la classification des œuvres exposées (shuppin burui mokuroku 出品部類目録) :

\section{二、書ヨ教育及學藝二編入シタルコト}

2. La calligraphie sera incluse dans la section "Education, Sciences et Arts » (Tōkyō-fu $1924: 13$ )

De fait, la calligraphie occupait, avec la gravure de sceaux, la douzième division (Daijūnirui sho oyobi tenkoku 第十二類 書及管刻) de la première section intitulée "Éducation, Sciences et Arts " (Daiichibu kyōiku oyobi gakugei 第壱部 教育及學藝), alors que la section consacrée aux beauxarts était la deuxième. La reconnaissance d'une appartenance artistique de 
la calligraphie ne fut donc définitivement admise qu'après la Deuxième Guerre mondiale, avec son inclusion comme cinquième section de l'Exposition japonaise des beaux-arts (Nitten 日展) en 1948.

\section{Conclusion}

Par le biais des associations, les calligraphes parvinrent à mettre en place un réseau qui pallia le rejet de leur discipline par les artistes et les théoriciens issus des beaux-arts. Michael Lucken dénombre, à l'ère Taishō, entre vingt et trente groupes artistiques importants actifs à Tokyo (Lucken 2008 : 133). Du côté des calligraphes, le bilan est certes plus modeste, mais fait apparaitre entre dix et quinze associations majeures (Uozumi 2001 : 122). Si les effets de la mise à l'écart de la calligraphie se faisaient encore sentir, la marginalisation radicale que l'on aurait pu attendre au début des années 1880 n'avait pas eu lieu.

Concernant la défense de la calligraphie (en tant que domaine) contre une supposée infériorité face aux beaux-arts, l'efficacité des associations et des expositions publiques qu'elles organisèrent est indéniable. En revanche, à l'échelle individuelle des artistes, leur rôle de garant dans l'évaluation des œuvres est plus discutable. Si le système de sélection sur critères techniques par un jury qualifié offrait davantage de garanties que les choix subjectifs des réunions de lettrés, la calligraphie n'en demeurait pas moins un "marché fermé ", au sens où l'entend le philosophe Yves Michaud (Michaud 2003), soit un marché dans lequel la valeur des œuvres n’est pas déterminée par le public, mais uniquement par les experts.

Dans le cas des associations, la fermeture du marché allait encore plus loin : la proportion des spécialistes habilités à évaluer les œuvres était extrêmement restreinte, limitée non seulement aux membres de l'association, mais même plus particulièrement à ceux suffisamment élevés dans la hiérarchie pour avoir fonction de jury. L'effet de fermeture fonctionnait également dans le sens inverse : seules pouvaient prétendre à évaluation les œuvres des membres inscrits. Les calligraphes se voyaient donc offrir un circuit d'exposition et de vente où la sélection des œuvres se limitait au cercle de l'association, et non à celui de la nation comme dans le cas des salons. Cet effet de fermeture se trouva renforcé par le fait que, de 1895 à 1912, les associations eurent l'apanage exclusif de l'organisation des expositions. 
Même après la réintégration de la calligraphie dans les salons artistiques, le lien ne fut pas coupé pour autant, puisque les jurys responsables de la section de calligraphie étaient la plupart du temps actifs dans le domaine des associations.

Si ces dernières évitèrent à la calligraphie une mise à l'écart trop prononcée, on peut en revanche affirmer que la mise en place d'une scène calligraphique nationale fut retardée d'autant par l'existence de ces réseaux, très efficaces, mais qui tendirent à fausser le libre jeu de l'activité artistique en fixant les critères d'appréciation, le goût dominant et le marché. 


\section{Bibliographie}

\section{Sources primaires}

KAWADA Takeshi 川田剛, 1892

«Sho wa bijutsu taru no setsu » 書は

美術たるの説 (Pourquoi la calligraphie est un des beaux-arts), Tōkyō gakushi kaiin zasshi 東京学士会院雑誌, 14 (9) : 337-373.

KOYAMA Shōtarō 小山正太郎, 1882a «Sho wa bijutsu narazu »書八美術ナラズ (La calligraphie ne fait pas partie des beaux-arts), Tōyō gakugei zasshi 東洋學藝雑誌, 8 : 172-175.

\section{KOYAMA Shōtarō,1882b}

«Sho wa bijutsu narazu » 書八美術ナラズ (La calligraphie ne fait pas partie des beaux-arts), Tōyō gakugei zasshi, 9:205-206.

\section{KOYAMA Shōtarō,1882c}

«Sho wa bijutsu narazu » 書八美術ナラズ (La calligraphie ne fait pas partie des beaux-arts), Tōyō gakugei zasshi, $10: 227-231$.

\section{Shodō 書道, 1938}

« Dai ikkai shumi no kōkankai : keika hōkoku » 第一回趣味の交換會——經過 報告 (Compte rendu de la première vente par adjudication des connaisseurs), Shodō, 7 (9) : 51.

\section{OKAKURA Tenshin 岡倉天心, 1882a}

" "Sho wa bijutsu narazu" no ron o yomu » 『書八美術ナラズ』ノ論习読ム (Lecture de l'essai « La calligraphie ne fait pas partie des beaux-arts »), Tōyō gakugei zasshi 東洋學藝雑誌, $11: 261-264$.

\section{OKAKURA Tenshin, 1882b}

" "Sho wa bijutsu narazu" no ron o yomu » 『書八美術ナラズ』ノ論习読ム (Lecture de l'essai « La calligraphie ne fait pas partie des beaux-arts »), Tōyō gakugei zasshi, $12: 296-297$.

\section{OKAKURA Tenshin,1882c}

" "Sho wa bijutsu narazu" no ron o yomu » 『書八美術ナラズ』ノ論习読ム (Lecture de l'essai « La calligraphie ne fait pas partie des beaux-arts »), Tōyō gakugei zasshi, 15 : 397-399.

ONOE Saishū 尾上柴舟, 1927

« Préface », in OKUYAMA Kindō

奥山錦洞, Nihon shodōshi 日本書道史 (Histoire de la calligraphie au Japon), Tokyo, Keibunsha 啓文社.

ŌTSUKI Joden 大槻如電, 1911 «Rikushokai sōgyō banashi » 六書会創業ばなし (Propos sur la création de l'Association des Six Styles »), Shoen 書苑, numéro inaugural, novembre.

TAKI Setsuan 瀧拙庵, 1926

《Shodō to bijutsu » 書道と美術 (La calligraphie et les beaux-arts), Kokka 國華, $422:$ 28-33.

\section{Tōkyō-fu 東京府, 1924}

Heiwa kinen Tōkyō hakurankai jimu $h o ̄ k o k u$ 平和記念東京博覧会事務報告 (Rapport administratif de l'Exposition 
de Tokyo pour la célébration de la Paix), Tokyo, Tōkyō-fu 東京府.

\section{Traductions}

\section{DIOT Rodolphe, 2009}

« La polémique Koyama-Okakura sur la calligraphie (1) », Jinbun kagaku $k e n k y \bar{u}$ 人文科学研究 (Recherches en sciences humaines), 5 : 95-106.

\section{DIOT Rodolphe, 2010}

«La polémique Koyama-Okakura sur la calligraphie (2) », Jinbun kagaku $k e n k y \bar{u}$ 人文科学研究 (Recherches en sciences humaines), $6: 253-262$.

GODDARD Timothy Unverzagt, 2012 "Reading 'Calligraphy is not art' (1882) », Review of Japanese Culture and Society, 24 : 168-175.

\section{Sources secondaires}

ANDŌ Tōseki 安藤搨石, 1964 Shodan hyakunen 書壇百年 (Cent ans du milieu de la calligraphie), Tokyo, Mokujisha 木耳社.

\section{CHARRIER Isabelle, 1999}

«La réaction nationaliste des années 1880 dans le milieu artistique : Ernest Fenollosa et Okakura Tenshin », in TSCHUDIN Jean-Jacques \& HAMON Claude (dir.), La Nation en marche. Études sur le Japon impérial de Meiji, Arles, Picquier, pp. 163-180.

\section{DOR Laïli, 2013}

«Discours de soi et discours de l'autre dans la polémique Koyama-Okakura sur la calligraphie », in BUTEL Jean-Michel et ANDRO Makiko (dir.), Japon Pluriel 9, Arles, Picquier, pp. 293-301.

\section{FOXWELL Chelsea, 2012}

"New Art and the Display of Antiquities in Mid-Meiji Tokyo ", Review of Japanese Culture and Society, décembre, 24 :

137-154.

\section{HEINICH Nathalie, 1993}

Du peintre à l'artiste. Artisans et académiciens à l'âge classique, Paris, Éditions de Minuit.

\section{HEINICH Nathalie, 1996}

Être artiste, Paris, Klincksieck.

\section{KITAZAWA Noriaki 北澤憲昭, [1989] 2010}

Me no shinden. "Bijutsu » juyōshi nōto 眼の神殿——美術」受容史ノート (Le sanctuaire du regard. Notes sur l'histoire de la réception des « beaux-arts »), Tokyo, Seiunsha 星雲社.

\section{KONDŌ Kōshi 近藤高史, 1991}

Meiji shodōshi yawa 明治書道史夜話 (Propos nocturnes sur la calligraphie de Meiji), Tōkyō, Geijutsu shinbunsha 芸術新聞社.

\section{LUCKEN Michael, 2008}

« L'évolution du statut de l'œuvre d'art au début du xxe siècle au Japon, à travers les expositions et les sociétés d'art », in BLANCHON Flora (dir.), La question de l'art en Asie orientale, Paris, Presses universitaires de ParisSorbonne, pp. 133-149. 
MARQUET Christophe, 1999

«Conscience patrimoniale et écriture de I'histoire de l'art national », in TSCHUDIN Jean-Jacques \& HAMON Claude (dir.), La Nation en marche, Arles, Picquier, pp. 143-162.

\section{MICHAUD Yves, 2003}

«Évaluations et prescriptions : en général et aujourd'hui en particulier », Revue francophone d'esthétique, 1 : 47-57.

\section{MITTEAU Arthur, 2013a}

«La critique de la peinture de lettrés par Fenollosa en 1882 : orientalisme latent ou stratégie assumée ? », in BUTEL JeanMichel \& ANDRO Makiko (dir.), Japon Pluriel 9, Arles, Picquier, pp. 283-291.

\section{MITTEAU Arthur, 2013b}

«L'universalisme de l'esthétique chez Okakura Kakuzō (dit Tenshin) et Ernest Fenollosa : critique et actualité », Ebisu, 50 : 95-133.

\section{PATIN Cléa, 2013}

« Le système des ventes d'œuvres d'art réservées aux professionnels au Japon (kōkankai) », Ebisu, 50 : 187-216.

SATŌ Dōshin 佐藤道信, 1999

Meiji kokka to kindai bijutsu. Bi no

seijigaku 明治国家と近代美術——美の

政治学 (L'État de l'ère Meiji et l'art moderne. Une politique du Beau), Tokyo, Yoshikawa Kōbunkan 吉川弘文館.

TAKAHASHI Toshirō 高橋利郎, 2011 Kindai nihon ni okeru sho e no manazashi 近代日本における書への眼差し (Regard sur la calligraphie dans le Japon moderne), Kyoto, Shibunkaku 思文閣.

\section{UENO Naoteru, 1958}

Japanese Arts and Crafts in the Meiji Era, Tokyo, Pan-Pacific Press.

\section{UOZUMI Kazuaki 魚住和晃}

\& HAGI Nobuo 萩信雄 (dir.), 2001 Shogaku kyoyō. Sho no rekishi to bunka 書学挙要——書の歴史と文化 (Précis d'études calligraphiques. La calligraphie, son histoire et sa culture), Kyoto, Geibun shoin 藝文書院.

URASAKI Eishaku 浦崎永錫, 1974 Nihon kindai bijutsu hattatsu shi 日本近代 美術発達史 (Histoire du développement des beaux-arts du Japon moderne), Tokyo, Tōkyō bijutsu 東京美術.

YOSHIDA Chizuko 吉田千鶴子, 2011 Nihon bijutsu no hakken「日本美術」の 発見 (La découverte des «beaux-arts japonais »), Tokyo, Yoshikawa Kōbunkan 吉川弘文館. 